Comparative and Functional Genomics

Comp Funct Genom 2005; 6: 159-169.

Published online in Wiley InterScience (www.interscience.wiley.com). DOl: 10.1002/cfg.469 \title{
Conference Paper
Plant reproductive genomics at the Plant and
Animal Genome Conference
}

\author{
Jim Leebens-Mack ${ }^{1}$, Douglas E. Soltis ${ }^{2}$ and Pamela S. Soltis ${ }^{3}$ \\ 'Department of Biology and Huck Institutes of the Life Sciences, Pennsylvania State University, University Park, PA I6802, USA \\ ${ }^{2}$ Department of Botany, University of Florida, Gainesville, FL 326II, USA \\ ${ }^{3}$ Florida Museum of Natural History, University of Florida, Gainesville, FL 3261 I, USA
}

*Correspondence to:

Jim Leebens-Mack, Department

of Biology and Huck Institutes of

the Life Sciences, Pennsylvania

State University, University Park,

PA 16802, USA.

E-mail: jleebensmack@psu.edu

Received: 31 January 2005

Accepted: 8 February 2005

Keywords: angiosperms; floral development; ABC model; comparative method

\section{Introduction}

Exciting advances in our understanding of plant reproductive biology are being spurred both by new research on established model systems and by the emergence of new models. Moreover, comparative analyses are placing knowledge derived from each of these systems into an evolutionary framework and thus providing insights into the processes responsible for the diversity of forms we see today. The number of species with large EST sets for floral transcriptomes is growing rapidly (Figure 1; http://pgn.cornell.edu/) and expression profiling of tagged genes is under way for many of these species. While there are still few tractable systems for experimental investigations of gene function, the development and refinement of virus-induced gene silencing (VIGS; reviewed in Benedito et al., 2004; Lu et al., 2004; Robertson, 2004) and targeting-induced local lesions genomes (TILLING; reviewed in Henikoff et al., 2004; Stemple, 2004) promise to expand the application of reverse genetics to non-model species. Experimental analysis in a broader range of plant lineages (Figure 1), including the basal-most eudicot lineages (Ranunculales), non-grass monocots, magnoliids and the basal-most angiosperm lineages (Amborellaceae and Nymphaeaceae; see Angiosperm Phylogeny Group, 2003), will help us understand how gene function has evolved since the most recent common ancestor of Arabidopsis and grain models (rice, maize, wheat and others) and all angiosperms.

The Plant Reproductive Genomics Workshop at the Plant Animal Genome Conference was organized to present recent advances in our comprehension of floral development and discuss how new genomic resources for an expanding set of plant species is continuing to deepen this understanding. The main topics covered in the workshop this year were floral diversity, variations of the classical $\mathrm{ABC}$ model of floral development, genetic analysis of reproductive traits, the regulation of flowering time and the origin and characterization of the floral transcriptome in the most recent common ancestor of all extant angiosperms. These topics were covered by 13 speakers in this workshop and by a number of speakers in other workshops at PAG XIII. The papers presented in the Plant Reproductive Genomics Workshop will be included in a symposium volume to be published early next year in the Advances in Botanical 


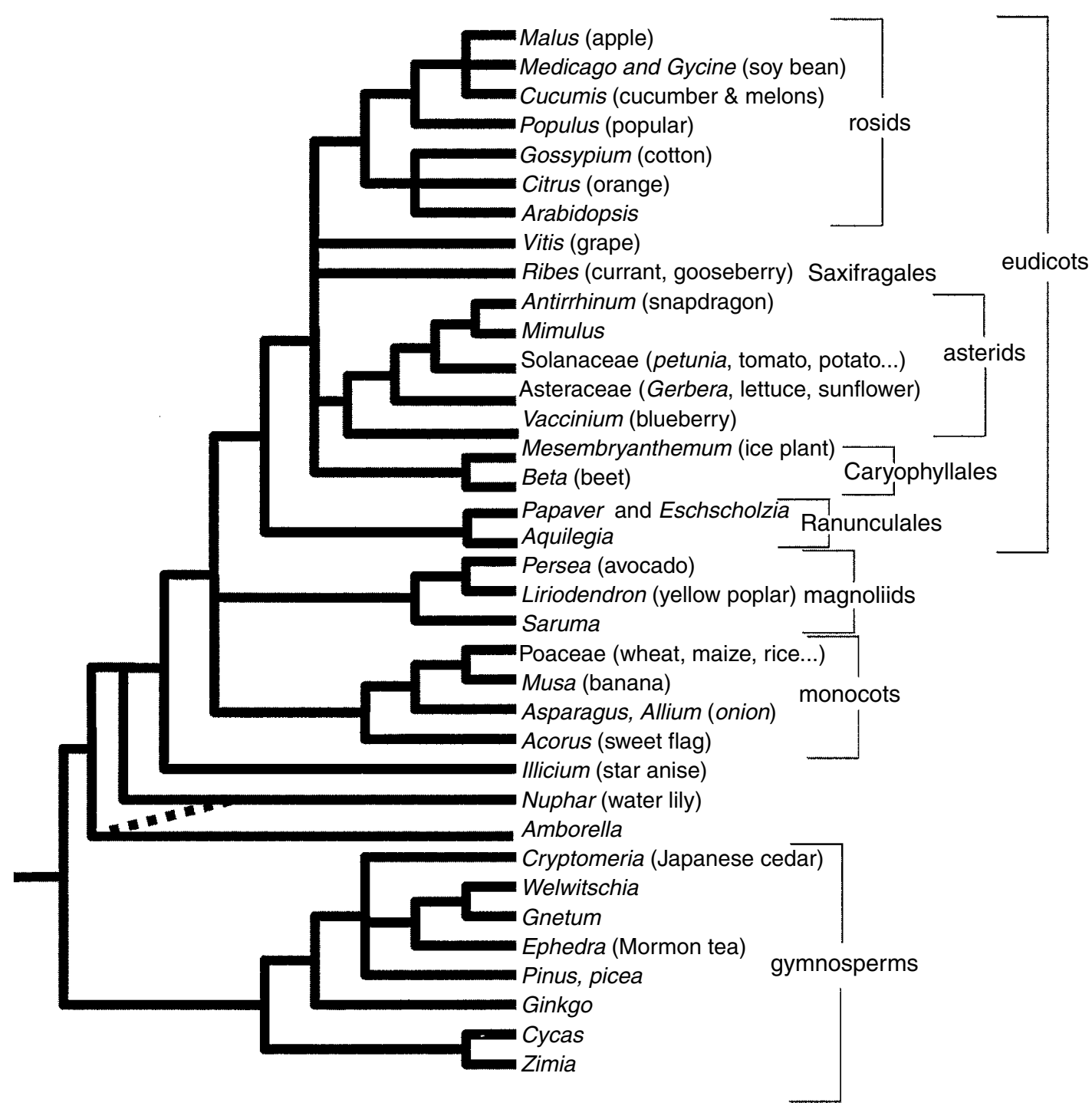

Figure I. Expressed sequence tags (ESTs) are being generated for a growing number of plant species representing major lineages of angiosperms and gymnosperms. The phylogeny shows the relationships among species for which large EST sets are available now or will be available in the near future. Multigene phylogenetic analyses provide varying levels of support for Amborella alone as sister to all other angiosperms (as indicated by the dashed line) or Amborella plus waterlilies, forming a clade that is sister to all other extant angiosperms (Zanis et al., 2002; Stefanovic et al., 2004)

Research series (Elsevier/Academic Press). Here we highlight some of the points presented in these talks.

\section{Floral diversity}

The current models used for studies of floral development exhibit a small fraction of the diversity of floral forms observed across the angiosperms. Peter Endress (University of Zurich) characterized the diversity of ontogenetic programs observed in floral development. Both Arabidopsis and grass flowers have their organs developing in well-defined whorls, whereas many species in the basal-most angiosperm lineages exhibit a spiral organization of floral organs with sequential, spiral development of perianth parts, then male organs, and finally female organs (Endress, 2001). This basic difference in phyllotaxy has implications for other aspects of floral development, e.g. the 
number of organs in a whorl may be limited by crowding. Synorganization of parts, as observed for eudicot species with fused corollas or syncarpous ovaries, may be achieved by congenital fusion in species with whorled floral organization, but this is very rare in species with spirally arranged floral organs. Postgenital coherence was important for the origin of angiospermy, as the carpel was probably sealed by secretion in the earliest angiosperms. Postgenital coherence through cuticular interdigitation, cellular interdigitation and postgenital fusion has also been important in the evolution of synorganization throughout angiosperm history, and these processes deserve more intensive investigation in diverse species with contrasting floral forms.

A striking variation on the development of flowers with whorled phyllotaxy is the existence of a ring meristem in some non-model lineages. The ring meristem is associated with a developmental decoupling of whorls with male and female organs. This decoupling has in turn extended the time of stamen initiation and allowed for the development of different types of stamens in some lineages. In the case of taxa such as Dillenia (Dilleniaceae), stamens are initiated centrifugally even after the gynoecium is completely closed, and sterile stamens formed later in development may act to increase pollinator activity (Endress, 1997). Again, this mode of floral development is not observed in current model systems and investigations of the changes in the molecular regulation of floral development in these systems would provide insights into the evolution of novel pollination systems.

\section{Variations on the $A B C(D E)$ model of floral organ initiation}

Over the last 15 years, forward and reverse genetic investigations focused largely on the core eudicots Antirrhinum majus, Arabidopsis thaliana and Petunia hybrida have formed a firm foundation for our understanding of the molecular mechanisms regulating flower development (reviewed in Irish, 2003; Jack, 2004; Kramer and Hall, 2005). In the early 1990s ground-breaking synthetic analyses of work on floral homeotic mutants in Antirrhinum and Arabidopsis culminated in the ABC model of floral development (Schwartz-Sommer et al., 1990; Coen and Meyerowitz, 1991). In this model (Figure 2), A-class genes specify development of sepals (first whorl) and petals (second whorl); B-class genes specify development of petals and stamens (third whorl); and C-class genes specify development of stamens and carpels (fourth whorl). The model also stipulates that A-class and C-class genes negatively regulate each other. Later experiments showed that a fourth class of genes is necessary for ovule development (Columbo et al., 1995), and a fifth class (E-class) works with the $\mathrm{ABC}$ genes to regulate floral organ initiation (Pelaz et al., 2000). All but one $(A P 2)$ of the genes implicated in the $\mathrm{ABC}(\mathrm{DE})$ model of floral development are transcription factors in the MIKC-type MADS box gene family (Figure 2). According to the quartet model (Theissen and Saedler, 2001), specific combinations of A-, B-, C- and E-class MADS proteins are hypothesized to form heterodimers or higher-order complexes that bind to CArG-box DNA motifs and regulate the development of specific floral organs (Theissen and Saedler, 2001).

Soon after the ABC model was proposed, evolutionary biologists started to reconstruct the relationships among MADS box genes (Doyle, 1994; Purugganan et al., 1995). This work has continued as more MADS genes have been identified (reviewed in Becker and Theissen, 2003) and comparative analyses are providing a deep understanding of how gene expression patterns and gene function have evolved through angiosperm history. A number of the speakers presented their latest

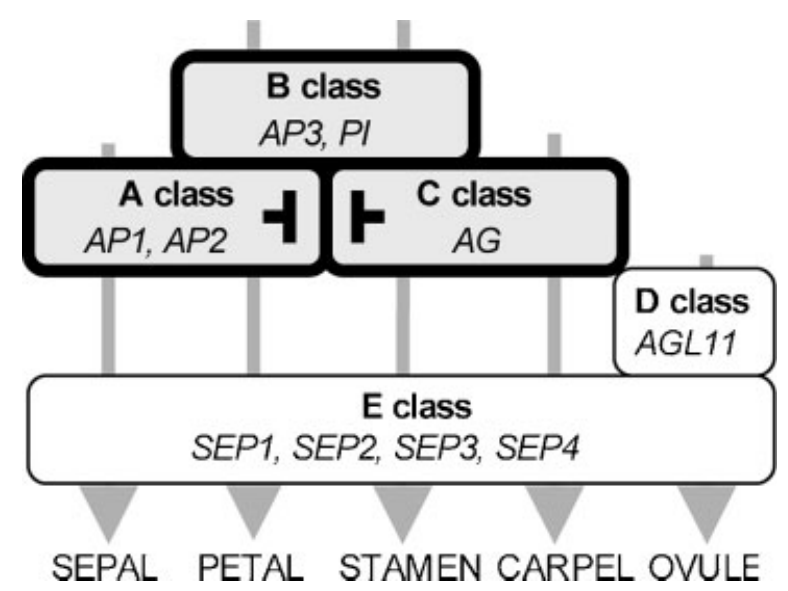

Figure 2. In the $A B C(D E)$ model of genetic control over floral development, distinct classes of genes specify organs in whorls I, 2, 3 and 4 of the flower and the ovules. In addition, class $A$ and class $C$ genes regulate each other antagonistically. The identities of these genes in Arabidopsis are shown in for each functional class 
research on MADS box gene expression and function. Gene duplication and shifting function were common themes in these talks. Aspects of the $\mathrm{ABC}(\mathrm{DE})$ model developed for the core eudicots seem to hold across all of the angiosperms, but the evolution of gene expression, and presumably function, is quite dynamic. Moreover, in many basal lineages of flowering plants, as well as basal eudicots, the $\mathrm{ABC}$ model has to be revised to accommodate flowers with spiral phyllotaxy or an undifferentiated perianth, rather than distinct sepals and petals.

Kerstin Kaufmann, a student in Guenter Theissen's lab (Friedrich Schiller University, Jena), presented their work on the physiochemical basis of the regulatory network of floral development hypothesized in the quartet model. The interaction of B-class genes regulating development of the petals and stamens has been studied most intensively in yeast two-hybrid experiments. In angiosperms, the B-class genes fall into two wellsupported clades, designated $D E F$ and $G L O$ after the Antirrhinum gene placed in each clade with the Arabidopsis genes AP3 and PI, respectively. The $D E F$ and $G L O$ clades arose from a common ancestor following a gene duplication that occurred some time after the divergence of the lineages leading to extant angiosperms and gymnosperms (Winter et al., 2002; Stellari et al., 2004; Kim et al., 2004). Protein interaction experiments and functional studies have shown that B-class function in most angiosperms studied to date requires both $D E F$ and $G L O$ orthologueues, which form a heterodimer in yeast two-hybrid experiments. The finding that the most closely related B-type gene in Gnetum (GGM2) is able to function as a homodimer led to the conclusion that obligate heterodimerization of B-type genes arose after the $D E F / G L O$ duplication (Winter et al., 2002). Recent yeast two-hybrid experiments, however, have shown that GGM2 is able to form heterodimers with several other MIKC-type MADS gene products identified in Gnetum. This raises the question of whether the ability of ancestral Bclass MADS proteins to form heterodimers was a precursor to obligate heterodimerization of MADS proteins for B-function in flowering plants.

Brendan Davies (University of Leeds) contrasted the function of C-class genes in Antirrhinum and Arabidopsis and touched on the utility of new genomic resources for studies of gene function in Antirrhinum. A gene duplication early in the history of core eudicots, before the divergence of rosids (including Arabidopsis) and asterids (including Antirrhinum), gave rise to two clades with genes that have been retained in both lineages. Davies and collaborators are comparing BAC clones containing the two Antirrhinum genes PLENA (PLE) and FARINELLI (FAR) with homologous regions in the Arabidopsis genome anchored by SHATTERPROOF1 (SHP1) and SHP2 and the C-class gene AGAMOUS (AG). Syntenic patterns are predicted based on recent phylogenetic analyses identifying AG and FAR as orthologues; and SHP1 and SHP2 are recent duplicates that are orthologues of PLE (Kramer et al., 2004).

The relationships among AG homologues in Arabidopsis and Antirrhinum somewhat surprising because the phenotypes of $a g$ and ple mutants are more similar to each other than they are to far and shp1 shp2 mutants, respectively. The $a g$ and ple mutants show homeotic conversion of reproductive tissues to perianth-like tissues, whereas far mutants form anthers with sterile pollen and intact carpels. The most apparent phenotype of shp1 shp2 double mutants is indehiscent fruits, although the SHP genes are also expressed in ovules. Analyses of far and ple single and double mutants suggest that $P L E$ is an upstream regulator of FAR and FAR is a repressor of PLE (Davies et al., 1999). As might be predicted, based on the phenotypes of far and ple, transformation experiments showed that FAR expression has a greater influence on male function, while PLE has a stronger influence on female function. Taken together, the data on C-type gene expression and function demonstrate different patterns of subfunctionalization and perhaps neofunctionalization (sensu Force et al., 1999) in Arabidopsis and Antirrhinum following a gene duplication event in a common ancestor.

Toby Kellogg (University of Missouri, St. Louis, MO) showed data on independent MADS gene duplication events in the grasses (Poaceae). Much of the work Kellogg and collaborators are doing on MADS gene evolution in the grasses is aimed at elucidating how gene duplications lead to functional and ultimately morphological diversification. Duplications of A-, C- and E-class genes just prior to diversification of the grass family have been followed by divergence in expression patterns. In all of these cases, however, duplicate genes have evolved under similar levels of constraint. There 
has been no detectable change in the ratio of nonsynonymous to synonymous nucleotide substitution rates following gene duplication.

Phylogenetic analysis of SEPALLATA-like (SEP) MADS genes shows independent duplication events in the monocots and eudicots. Whereas the Efunction of SEP1, SEP2 and SEP3 seems largely redundant in Arabidopsis, expression patterns vary among many duplicates in the grass family. Investigations of one of these duplicates show that differences in the expression patterns observed among distinct grass lineages are associated with differences in spikelet development. LEAFY HULL STERILE1 (LHS1) orthologues are expressed in the reproductive meristem of all grasses. However, as development proceeds in panicoid species (e.g. maize, sorghum, pearl millet) with two flowers per spikelet and top-down maturation, LHS1-like gene expression is limited to the upper floret. While the same pattern of expression is observed in development of the three-flowered rice spikelets, lineages with many flowers per spikelet and bottom-up maturation show gradual reduction in LHS1-like gene expression through development of all florets (Malcomber and Kellogg, 2004). Adding to this diversity, organ-specific expression patterns within flowers vary among species with both top-down and bottom-up spikelet development. The independent diversification of multiple MADS gene lineages early in the history of the Poaceae raises the possibility of manifold diversification in gene interaction partnerships and function. Perhaps this diversity at the molecular level has played a role in generating morphological variation and the impressive species diversity observed in the grass family.

The number of grass species is topped only by the species richness observed within the Orchidaceae and Asteraceae. Teemu Teeri (University of Helsinki) presented the work he and his collaborators have done on MADS gene function in Gerbera hybrida (Asteraceae). Like grasses, members of the sunflower family have flowers that are quite distinct from those of Arabidopsis, Antirrhinum or Petunia. The flower head (inflorescence) of Gerbera includes ray flowers with aborted stamens and disk flowers with reduced petals, and all flowers have specialized pappus bristles derived from sepals, fused petals and anthers, and inferior ovaries.

The B- and C-function homologues in Gerbera are placed with their Antirrhinum and Arabidopsis counterparts in MADS gene phylogenies. The Gerbera SEP-like genes, however, have very diverse functions (Uimari et al., 2004). Unlike the redundant expression patterns of SEP1, SEP2 and SEP3 in whorls 2, 3 and 4 of Arabidopsis flowers, distinct Gerbera SEP homologues show evidence of subfunctionalization. Expression of GRCD1 is required for stamen development, while GRCD2 is needed for proper initiation of carpels. Downregulation of $G R C D 2$ also results in floral reversion, producing flowers within the carpels! In addition, reduced $G R C D 2$ expression leads to larger inflorescences (capitula). These observations indicate that GRCD2, like SEP4 in Arabidopsis (Ditta et al., 2004), is involved in the regulation of reproductive meristem determinacy.

Vivian Irish (Yale University) and co-workers have initiated analyses to examine the extent to which gene duplication, regulatory diversification and differences in protein interactions have been important in modifying the roles of MADS box genes during the evolution of flowering plants. It is noteworthy that the occurrence of several key duplications of floral organ identity genes seems to correspond with the origin of core eudicots (e.g. Irish, 2003; Litt and Irish, 2003; Kramer et al., 2004; Stellari et al., 2004; Kim et al., 2004; Zahn et al., 2005).

In efforts to attain a more complete understanding of the role of duplicated MADS genes, the Irish lab has developed a protocol for rapidly identifying all MADS box genes from a given species. Reconstruction of phylogenetic relationships among MADS genes is providing a historical context for the results of functional analyses. For example, tomato MADS box genes and their relationship to well characterized MADS genes from model species have been identified and current studies are elucidating the function of these genes in tomato flower and fruit development (e.g. DiMartino and Irish, in preparation).

Molecular genetic investigations have provided insights into the molecular evolution and function of various MADS duplicates. For example, considering the duplication of the B-class gene AP3, a divergent C-terminus motif in the euAP3 lineage appears to have arisen via a frameshift mutation (Vandenbussche et al., 2003). The paleoAP3 gene may have only specified stamen identity and not petal identity, as euAP3 does in core 
eudicots (Lamb and Irish, 2003; but see Whipple et al., 2004).

Irish stressed the importance of obtaining more MADS data, including functional analyses, for non-model angiosperms (similar statements were echoed later by Kramer and Soltis). Poppy is proposed as a potential new model, representing the phyogenetically pivotal basal eudicots. However, Irish et al. have encountered problems with transformation in poppy. As result, they have been developing alternative methods to carry out functional analyses in this and other non-model basal eudicots. One method that shows great promise in poppy is the use of VIGS using tobacco rattle virus (Liu et al., 2004).

Elena Kramer (Harvard University) continued the theme of modified $\mathrm{ABC}(\mathrm{DE})$ models to explain variation in floral form. Her presentation focused on recent work on duplicated B-function homologues in Ranunculaceae, a clade of basal eudicots that exhibits a great deal of variation in floral organization, with the intent of relating differential expression of paralogues to organ differentiation within a flower and to differences in floral structure among species. She began her presentation by asserting that, given the range of floral morphologies across the angiosperms and our currently rather limited understanding of the genes controlling perianth identity, there are multiple petal identity programs at work in different groups of angiosperms. Furthermore, some groups, notably Ranunculaceae, have an abundance of B-function homologues that are not due to increases in ploidy and that may play roles in floral differentiation in the family (e.g. Kramer et al., 2003). Based on her lab's work on Aquilegia (columbine), it appears that multiple AP3 paralogues may each have distinct functions in perianth differentiation. For example, the PI homologue and one of the AP3 homologues, AP3-1, have expression patterns consistent with PI and AP3 in Arabidopsis. AP32 is expressed later in the petals and for longer duration in the stamens, whereas AP3-3 expression is restricted to petals and may play a role in the development of the spurred petals and nectaries typical of Aquilegia. Later expression of PI in the outer perianth may confer petaloid characteristics to these organs, rather than organ identity per se. A combination of 'sliding boundaries' (Kramer et al., 2003) of duplicate genes and temporal variation in expression may be responsible for perianth differentiation in Aquilegia. Using yeast2-hybrid studies, Kramer found some interactions between AP3-2 and AP3-3, but most heterodimers are between $P I$ and $A P 3$ subunits. Future analysis of numerous homeotic horticultural mutants, 35000 ESTs and microarrays should help to refine the petal identity program(s) at work in Aquilegia and address the possible roles of sub- and neofunctionalization of duplicate genes in shaping floral morphology.

Pamela Soltis (University of Florida) presented a modified $\mathrm{ABC}(\mathrm{DE})$ model of floral organ identity for basal angiosperms characterized by an undifferentiated perianth and gradual intergradation of floral organs. The model is based on studies of expression of homologues of the ABCE-function genes of Arabidopsis in a set of basal angiosperms: Amborella trichopoda, Nuphar advena and Illicium floridanum, representing the three basal-most lineages of extant angiosperms (Figure 1), and three representatives of the magnoliid clade, Magnolia grandiflora, Eupomatia bennettii (and E. laurina) and Asimina longifolia. Of these species, only A. longifolia has a perianth that is differentiated into distinct sepals and petals, and Eupomatia lacks a perianth entirely. Antirrhinum majus was used as a point of reference. Homologues of all classes of $\mathrm{ABC}(\mathrm{DE})$ genes have been reported from basal angiosperms (e.g. Kim et al., 2004, 2005; Kramer et al., 1998, 2003; Litt and Irish, 2003; Stellari et al., 2004), and Soltis reported that most classes of these genes have been found in the species chosen for study. Using a combination of relative quantitative RT-PCR, real-time PCR and in situ hybridization studies, she reported consistent patterns of gene expression for floral MADS box homologues of $A P 1, A P 3, P I, A G$ and $S E P$, despite differences in sensitivities of the techniques and in stages of floral development assayed. In general, floral MADS box genes are expressed in organs predicted by the ABC(DE) model of Arabidopsis and Antirrhinum, but expression, particularly of B-function homologues, is broader than predicted. The sole exception to this pattern is A. longifolia, with its sepaloid outer perianth whorl and two inner whorls of petaloid perianth parts, in which the $P I$ homologue is restricted to petals and stamens and the AP3 homologue is expressed at only low levels in the sepals. This pattern of expression is very similar to that of core eudicots characterized by a differentiated, whorled perianth and suggests 
that the same mechanism that evolved in eudicots appears to have arisen in this magnoliid.

Based on these results, Soltis and collaborators proposed that the ancestral angiosperms most likely exhibited broad patterns of MADS box gene expression across the floral meristem. Restriction of gene expression to more localized areas of the meristem may have led to the origin of whorled flowers with distinct sepals and petals - those flowers typical of most eudicots. Furthermore, broad - and intergrading - expression of key floral regulators corresponds to patterns of morphological intergradation of adjacent floral organs in Amborella (e.g. Buzgo et al., 2005) and likely other species. The 'fading borders' model, in which expression of each regulator is weaker at the outer and inner edges of its zone of activity, may explain the gradual transitions of floral organs - from bracts to sepaloid perianth to petaloid perianth to stamens, staminodes and perhaps carpels - often observed in basal angiosperms.

\section{Quantitative genetics and floral form}

John Willis (Duke University) reported on work he and colleagues have been doing on the Мimulus guttatus complex (Phrymaceae, formerly Scrophulariaceae). Adding to the quantitative genetic analyses of differences in floral traits and mating systems and the nature of the species boundary between the outcrossing $M$. guttatus and the selfing species, M. nasutus, large sets of BAC end sequences and ESTs are being used to increase the density of the genetic map for the M. glutattus complex. With its small genome size $(\sim 425 \mathrm{Mb} / 1 \mathrm{C})$, growing set of genomic resources, rapid regeneration time (2 months) and morphological and ecological diversity, Mimulus is a strong candidate for whole genome sequencing in the near future. Genetic mapping work has already identified 24 QTLs for reproductive traits in Mimulus (Fishman et al., 2002) and led to the localization of a region responsible for true meiotic drive in hybrid crosses (Fishman and Willis, 2004). The QTL analysis shows that many genes of small effect can influence floral form and outcrossing rate. Therefore, reverse genetics involving a few candidate genes will not provide a complete picture of the genes influencing variation in flower shape and size. Quantitative analysis of variation in floral colour patterning among Mimulus species is under way. All of this work is providing insights into the molecular basis of reproductive diversity within and among species.

In the International Grass Genome Initiative Workshop, Kellogg presented the complementary QTL and candidate gene analyses of inflorescence branching in foxtail and green millet. This work, led by Andrew Doust (Doust et al., 2004, 2005), revealed co-localization of candidate genes with QTL influencing inflorescence branch number and density, spikelet number and bristle number. For example, a marker for the $L E A F Y$ orthologue mapped in close proximity to a QTL for primary branch number and density. No genomic resources other than the genetic map were available for the two millet species compared in this study, but Doust, Kellogg and collaborators were able to leverage the sequence data available for related model grain species and uncover important aspects of the genetic basis of differences in the inflorescence architectures of two millet species.

\section{Regulation of flowering time}

Michael Purugganan (North Carolina State University) discussed how microevolutionary analyses of population variation in Arabidopsis thaliana are elucidating how natural selection across a latitudinal gradient is driving the evolution of the flowering time regulatory network. Purugganan and colleagues are in the process of testing for clinal variation in all genes known to be in the flowering time regulatory network. To date, analyses of FLOWERING LOCUS C (FLC) and active forms of the upstream regulator FRIGIDA (FRI) are exhibiting latitudinal clines in haplotype frequencies (Stinchcombe et al., 2004; Caicedo et al., 2004). FLC expression, which represses flowering, is upregulated by $F R I$ and downregulated by signals from the autonomous and vernalization flowering time pathways. Significant linkage disequilibrium between $F R I$ and $F L C$ haplotypes, together with the results of field experiments showing significant effects on size of flowering plants for both loci and their interaction, provide strong evidence for the influence of natural selection on the epistatic interaction between FRI and FLC. This exciting research is showing how natural selection acting on the initiation of reproductive development results in intraspecific variation in the genetic interactions 
regulating flowering time. Experimental tests of alternative hypotheses for the molecular mechanisms behind this response are under way.

New findings on the control of flowering time were also presented by J. Chris Pires (University of Wisconsin) in the Brassicas Workshop and Masahiro Yano (National Institute of Agrobiological Sciences, Japan) in his plenary address. Pires presented work on the segregation and expression of FLC genes in synthetic Brassica polyploids (Schranz and Osborn, 2004; Pires et al., 2004). Flowering time in synthetic polyploids was strongly influenced by the source of expressed FLC homologues at three loci. As might be expected, based on flowering time differences between the two diploid parental species, expression of B. oleracea homologues was greater in late-flowering lines while $B$. napus homologues were more highly expressed in early-flowering lines. Gene expression levels were influenced by chromosomal rearrangements and epigenetic gene silencing.

$F L C$ is a MADS box gene, but to date, no closely related homologues have been observed in species of Brassicaceae. The regulation of flowering time in rice differs from Arabidopsis and Brassica in this regard and many others. Masahiro Yano presented the large body of work from his lab on the genetics of photoperiodic control of flowering time in rice (e.g. Kojima et al., 2002; Izawa et al., 2003; Doi et al., 2004). Comparisons of QTL maps and gene expression in nearly isogenic lines (NILs) with naturally occurring loss-of-function alleles are elucidating gene interactions with a regulatory network and the molecular basis of natural flowering time among rice lines. Phylogenies for gene families including flowering time regulators from rice and Arabidopsis show that gene duplications in the ancestors of both species and subsequent evolution in gene function have contributed to differences in the control of flowering time in these two species.

\section{Characterizing the most recent common ancestor of all living angiosperms}

The evolutionary processes responsible for the origin and rapid radiation of flowering plants has long been a major question in plant evolution, famously called an 'abominable mystery' by Darwin. Throughout the 1900s, the large morphological gap between flowers and potential gymnosperm relatives and possible ancestors permitted a multitude of widely different and mutually incompatible hypotheses to be entertained. The recent consensus on relationships among basal angiosperms, and the demonstration that living gymnosperms are monophyletic (so none are particularly closely related to angiosperms), now substantially narrow the range of reasonable models of angiosperm origins. Our changing views on the origin of the flowering plants are not only influenced by prevailing views and assumptions, but a renewed appreciation of old, long-ignored developmental literature has also been important. Recent evidence from genes that control development has already led to theories that are more specific and more testable than older theories.

Michael Frohlich (Natural History Museum, London) discussed prospects for ongoing and future research on the origin of the flower and flowering plants. The most recent theories focus on the origin of flower bisexuality from gymnosperm ancestors that produced separate male and female structures. A recent proposal for the origin of the flower is the Mostly Male Theory (Frohlich and Parker, 2000). In brief, gymnosperms generally have two copies of the $L F Y$ gene, referred to as the 'needle' and 'leaf' families. The 'needle' (NEEDLY) paralogue $\left(L F Y_{f}\right)$ is expressed only in early-developing female reproductive structures, whereas the 'leaf' paralogue $\left(L F Y_{m}\right)$ is expressed in male reproductive structures. The $L F Y$ duplication in gymnosperms may have accompanied or perhaps facilitated the specialization of separate male and female reproductive structures. In the Mostly Male model, $L F Y$ is a trigger for the origin of the flower. Angiosperms have apparently lost the female-specifying 'needle' paralogue and retained the male-related 'leaf' paralogue. Frohlich and Parker (2000) suggested that developmental control of floral organization derives more from systems operating in the male reproductive structures of the gymnosperm ancestor of angiosperms than from the female reproductive structures; hence 'mostly male.' In the Mostly Male Theory, ovules are considered ectopic in origin on male reproductive structures. Supporting this possibility is old evidence in the literature for ectopic expression in weird places in plants, such as ovules on leaves in Ginkgo.

Albert et al. (2002) provided an alternative to the Mostly Male Theory. Their model assumes that pleiotropic interactions between $L F Y_{m}$ and 
$L F Y_{f}$ were critical for stabilizing the retention of these two genes in gymnosperms, and suggests that disruption of this delicate balance between the two $L F Y$ genes occurred in an ancestor of modern angiosperms. This ancestral taxon might have had unisexual flowers together on the same plant, or might have been loosely bisexual. $L F Y_{f}$ would then have been lost through selection for an integrated bisexual reproductive axis.

Views on morphology can also change our perspective on the origin and diversification of the flower. Frohlich provided an example based on the perianth of the water lilies Nuphar and Nymphaea (Nymphaeaceae). The distinction between sepals and petals in Nuphar and Nymphaea was traditionally based on morphology, colour differences and glands. But Frohlich showed that in water lilies, sepal vs. petal identity also depends on position, age of organ, environment (light, physical contact) and interactions among these factors; furthermore, outer organs shield inner organs and the latter become petal-like. However, aspects of 'sepalness' and 'petalness' are not fixed to organs until later in development. In fact, sepal-like vs. petal-like is a difficult distinction (the terms usually refer to whole organs) but Frohlich showed that they may refer, in basal angiosperms, to organ segments, as in Nuphar, where part of a single organ may look like a sepal while other parts look like a petal - this is a new twist to looking at morphology and organ identity. Distinct sepal and petal developmental programs arose early in angiosperm history but may not have been clearly anchored to individual organs in early angiosperms. This anchoring did not really arise until the evolution of eudicots - further evidence of the floral developmental flexibility of basal angiosperms.

Claude dePamphilis (Penn State University) and collaborators involved in the Floral Genome Project (http://www.floralgenome.org/) are using a combination of EST sequencing and gene expression analyses to characterize the minimal genetic toolbox for floral development that must have been present in the last common ancestor of all extant angiosperms. Two very interesting observations are coming from genomic analyses of large EST sets generated for basal angiosperms Amborella trichopoda and Nuphar advena, magnoliids Persea americana (avocado) and Liriodendron tulipifera (tulip poplar), and representatives of the basal-most monocot and eudicot lineages,
Acorus americanus and Eschscholzia californica, respectively (Figure 1). Adding to a growing body of evidence that polyploidization has been common in angiosperm history, genome-wide duplication events are evident in analyses of all of the listed EST sets, with the exception of Amborella. At the same time, estimates of the number of genes expressed in developing flower buds do not vary appreciably across species. Despite the incomplete sampling inherent in EST sequencing projects, phylogenetic analyses of gene families, including known regulators of flowering time and floral organ identity in rice and Arabidopsis, showed that the last angiosperm common ancestor had a diverse set of potential regulators. The placement of basal angiosperm genes in the phylogenies with rice and Arabidopsis genes is helping to resolve the timing of gene duplications relative to the most recent common ancestor of monocots and eudicots.

\section{Comparative plant genomics}

Throughout the Plant Reproductive Genomics Workshop and the entire Plant and Animal Genome Conference, many and perhaps most talks included comparative analyses that drew inferences from cross-species similarities or differences in gene content, expression or function. The increasing base of species for which these data are available is improving our ability to draw accurate conclusions about the evolutionary processes that have generated the biodiversity we observe around us. Jim Leebens-Mack (Pennsylvania State University) ended the Workshop with some examples of how phylogenetically-based comparative genomics provides powerful tools for learning about individual plant species or groups of related species. A comparison of Arabidopsis, Populus, Medicago and rice genomic sequences in regions surrounding the typically single-copy gene $L E A F Y$ showed that the promoter of the Medicago orthologue has lost a conserved regulatory motif that is conserved between Arabidopsis and Populus. Cluster analysis of the predicted rice and Arabidopsis proteomes reveals over 4000 single-copy Arabidopsis genes with no orthologues in rice (http://www.PlantTribes.org). Patrick Zheng, a postdoc in David Openheimer's lab (University of Florida), is analysing T-insertion mutants for 
some of these genes and finding that reducedfunction mutants have highly compromised phenotypes. Orthologues of these genes were found in some of the Floral Genome Project EST sets, including the monocots asparagus and yucca, but not in the massive EST database available for members of the grass family. This work is identifying genes that are critical to development in Arabidopsis but were lost at some time before the diversification of grain crops.

As more expression and gene function data are being generated for a broader collection of plant species, phylogenetically-based comparative analyses will promote a deeper understanding of the molecular basis of variation in the development of flowers and other traits. Such advances will require careful staging of development in order to make appropriate cross-species comparisons (Buzgo et al., 2004). Improved phylogenetic inferences of gene family evolution will require increased sampling of genes from species representing key lineages in the angiosperm phylogeny (Soltis et al., 2004) and improved methods of automated sequence alignment for large and diverse gene families. Finally, phylogeneticallybased methods have not yet been developed for identifying concerted changes in gene expression across the 100 s of gene families represented in microarray studies. Resolving these issues in the near future will be a major breakthrough for the field of functional genomics.

\section{Acknowledgements}

We thank all participants in the Plant Reproductive Genomics Workshop for a truly stimulating series of talks. Thanks go to Hong Ma and Günter Theissen for many discussions on the genetic control of floral development. Sangtae Kim provided Figure 2 depicting the ABC(DE) model. Travel costs for the workshop were funded in large part through National Science Foundation Plant Genome Grant, DBI-0115684, in support of the Floral Genome Project.

\section{References}

Albert VA, Oppenheimer DG, Lindqvist C. 2002. Pleiotropy, redundancy and the evolution of flowers. Trends Plant Sci 7: 297-301.

APGII. 2003. An update of the Angiosperm Phylogeny Group classification for the orders and families of flowering plants: APG II. Bot J Linn Soc 141: 399-436.
Becker A, Theissen G. 2003. The major clades of MADS-box genes and their role in the development and evolution of flowering plants. Mol Phylogenet Evol 29: 464-489.

Benedito VA, Visser PB, Angenent GC, Krens FA. 2004. The potential of virus-induced gene silencing for speeding up functional characterization of plant genes. Genet Mol Res 3: 323-341.

Buzgo M, Soltis DE, Soltis PS, Ma H. 2004. Towards a comprehensive integration of morphological and genetic studies of floral development. Trends Plant Sci 9: 164-173.

Buzgo M, Soltis PS, Soltis DE. 2005. Floral developmental morphology of Amborella trichopoda (Amborellaceae). Int J Plant Sci 165: 925-947.

Caicedo AL, Stinchcombe JR, Olsen KM, Schmitt J, Purugganan MD. 2004. Epistatic interaction between Arabidopsis FRI and FLC flowering time genes generates a latitudinal cline in a life history trait. Proc Natl Acad Sci USA 101: 15 670-15 675-.

Coen ES, Meyerowitz EM. 1991. The war of the whorls: genetic interactions controlling flower development. Nature 353: 31-37.

Columbo L, Franken J, Koetje E, et al. 1995. The petunia MADS box gene FBP11 determines ovule identity. Plant Cell 7: 1859-1868.

Davies B, Motte P, Keck E, et al. 1999. PLENA and FARINELLI: redundancy and regulatory interactions between two Antirrhinum MADS-box factors controlling flower development. EMBO J 18: 4023-4034.

Ditta G, Pinyopich A, Robles P, Pelaz S, Yanofsky MF. 2004. The SEP4 gene of Arabidopsis thaliana functions in floral organ and meristem identity. Curr Biol 14: 1935-1940.

Doi K, Izawa T, Fuse T, et al. 2004. Ehd1, a B-type response regulator in rice, confers short-day promotion of flowering and controls FT-like gene expression independently of Hd1. Genes Dev 18: 926-936.

Doust AN, Devos KM, Gadberry MD, Gale MD, Kellogg EA. 2004. Genetic control of branching in foxtail millet. Proc Natl Acad Sci USA 101: 9045-9050.

Doust AN, Devos KM, Gadberry MD, Gale MD, Kellogg EA. 2005. The genetic basis for inflorescence variation between foxtail and green millet (Poaceae). Genetics (in press).

Doyle JJ. 1994. Evolution of a plant homeotic multigene family: toward connecting molecular systematics and molecular developmental genetics. Syst Biol 43: 307-328.

Endress PK. 1997. Relationships between floral organization, architecture, and pollination mode in Dillenia (Dilleniaceae). Plant Syst Evol 206: 99-118.

Endress PK. 2001. The flowers in extant basal angiosperms and inferences on ancestral flowers. Int J Plant Sci 162: 1111-1140.

Fishman L, Kelly AJ, Willis JH. 2002. Minor quantitative trait loci underlie floral traits associated with mating system divergence in Mimulus. Evolution 56: 2138-2155.

Fishman L, Willis JH. 2004. A novel meiotic drive locus nearcompletely distorts segregation in Mimulus (monkeyflower) hybrids. Genetics (in press).

Force A, Lynch M, Pickett FB, Amores A, Yan YL, Postlethwait J. 1999. Preservation of duplicate genes by complementary, degenerative mutations. Genetics 151: 1531-1545.

Frohlich MW, Parker DS. 2000. The mostly male theory of flower evolutionary origins: from genes to fossils. Syst Bot 25: $155-170$. 
Henikoff S, Till BJ, Comai L. 2004. TILLING. Traditional mutagenesis meets functional genomics. Plant Physiol $\mathbf{1 3 5}$ 630-636.

Irish VF. 2003. The evolution of floral homeotic gene function. Bioessays 25: 637-646.

Irish VF, Benfey PN. 2004. Beyond Arabidopsis. Translational biology meets evolutionary developmental biology. Plant Physiol 135: 611-614.

Izawa T, Takahashi Y, Yano M. 2003. Comparative biology comes into bloom: genomic and genetic comparison of flowering pathways in rice and Arabidopsis. Curr Opin Plant Biol 6: $113-120$.

Jack T. 2004. Molecular and genetic mechanisms of floral control. Plant Cell 16: S1-17.

Kim S, Koh J, Ma H, et al. 2005. Sequence and expression studies of A-, B-, and E-class MADS-box genes in Eupomatia (Eupomatiaceae): support for the bracteate origin of the calyptra. Int J Plant Sci (in press).

Kim S, Yoo M-J, Albert VA, et al. 2004. Phylogeny and diversification of B-function MADS-box genes in angiosperms: evolutionary and functional implications of a 260-million-yearold duplication. Am J Bot 91: 2102-2118.

Kojima S, Takahashi Y, Kobayashi Y, et al. 2002. Hd3a, a rice orthologue of the Arabidopsis FT gene, promotes transition to flowering downstream of Hd1 under short-day conditions. Plant Cell Physiol 43: 1096-1105.

Kramer EM, Dorit RL, Irish VF. 1998. Molecular evolution of genes controlling petal and stamen development: duplication and divergence within the APETALA3 and PISTILLATA MADSbox gene lineages. Genetics 149: 765-783.

Kramer EM, Hall JC. 2005. Evolutionary dynamics of genes controlling floral development. Curr Opin Plant Biol 8: 13-18.

Kramer EM, Jaramillo MA, Di Stilio VS. 2004. Patterns of gene duplication and functional evolution during the diversification of the AGAMOUS subfamily of MADS box genes in angiosperms. Genetics 166: 1011-1023.

Kramer EM, Stilio VSD, Schluter PM. 2003. Complex patterns of gene duplication in the APETALA3 and PISTILLATA lineages of the Ranunculaceae. Int J Plant Sci 164: 1-11.

Lamb RS, Irish VF. 2003. Functional divergence within the APETALA3/PISTILLATA floral homeotic gene lineages. Proc Natl Acad Sci USA 100: 6558-6563.

Litt A, Irish VF. 2003. Duplication and diversification in the APETALA1/FRUITFULL floral homeotic gene lineage: implications for the evolution of floral development. Genetics 165: 821-833.

Liu Y, Nakayama N, Schiff M, et al. 2004. Virus induced gene silencing of a DEFICIENS orthologue in Nicotiana benthamiana. Plant Mol Biol 54: 701-711.

Malcomber ST, Kellogg EA. 2004. Heterogeneous expression patterns and separate roles of the SEPALLATA gene LEAFY HULL STERILE1 in grasses. Plant Cell 16: 1692-1706.

Pelaz S, Ditta GS, Baumann E, Wisman E, Yanofsky MF. 2000 $\mathrm{B}$ and $\mathrm{C}$ floral organ identity functions require SEPALLATA MADS-box genes. Nature 405: 200-203.
Pires JC, Zhao J, Schranz ME, et al. 2004. Flowering time divergence and genomic rearrangements in resynthesized Brassica polyploids (Brassicaceae). Biol J Linn Soc 82: 409-700.

Purugganan MD, Rounsley SD, Schmidt RJ, Yanofsky MF. 1995. Molecular evolution of flower development: diversification of the plant MADS-box regulatory gene family. Genetics 140: 345-356.

Robertson D. 2004. VIGS vectors for gene silencing: many targets, many tools. Annu Rev Plant Biol 55: 495-519.

Schranz ME, Osborn TC. 2004. De novo variation in life-history traits and responses to growth conditions of resynthesized polyploid Brassica napus (Brassicaceae). Am J Bot 91: $174-183$.

Schwarz-Sommer Z, Huijser P, Nacken W, Saedler H, Sommer H. 1990. Genetic control of flower development by homeotic genes in Antirrhinum majus. Science 250: 931-936.

Soltis DE, Albert VA, Savolainen V, et al. 2004. Genome-scale data, angiosperm relationships, and 'ending incongruence': a cautionary tale in phylogenetics. Trends Plant Sci 9: 477-483.

Stefanovic S, Rice DW, Palmer JD. 2004. Long branch attraction, taxon sampling, and the earliest angiosperms: Amborella or monocots? BMC Evol Biol 4: 35.

Stellari GM, Jaramillo MA, Kramer EM. 2004. Evolution of the APETALA3 and PISTILLATA lineages of MADS-boxcontaining genes in the basal angiosperms. Mol Biol Evol 21: $506-519$.

Stemple DL. 2004. TILLING - a high-throughput harvest for functional genomics. Nature Rev Genet 5: 145-150.

Stinchcombe JR, Weinig C, Ungerer M, et al. 2004. A latitudinal cline in flowering time in Arabidopsis thaliana modulated by the flowering time gene FRIGIDA. Proc Natl Acad Sci USA 101: 4712-4717.

Theissen G, Saedler H. 2001. Plant biology. Floral quartets. Nature 409: 469-471.

Uimari A, Kotilainen M, Elomaa P, et al. 2004. Integration of reproductive meristem fates by a SEPALLATA-like MADS-box gene. Proc Natl Acad Sci USA 101: 15817-15 822-.

Vandenbussche M, Theissen G, Van de Peer Y, Gerats T. 2003. Structural diversification and neo-functionalization during floral MADS-box gene evolution by C-terminal frameshift mutations. Nucleic Acids Res 31: 4401-4409.

Whipple CJ, Ciceri P, Padilla CM, et al. 2004. Conservation of B-class floral homeotic gene function between maize and Arabidopsis. Development 131: 6083-6091.

Winter KU, Weiser C, Kaufmann K, et al. 2002. Evolution of class B floral homeotic proteins: obligate heterodimerization originated from homodimerization. Mol Biol Evol 19: 587-596.

Zahn LM, Kong H, Leebens-Mack J, et al. 2005. The evolution of the SEPALLATA subfamily of MADS-box genes: a pre-angiosperm origin with multiple duplications throughout angiosperm history. Genetics (in press).

Zanis MJ, Soltis DE, Soltis PS, Mathews S, Donoghue MJ. 2002. The root of the angiosperms revisited. Proc Natl Acad Sci USA 99: 6848-6853. 

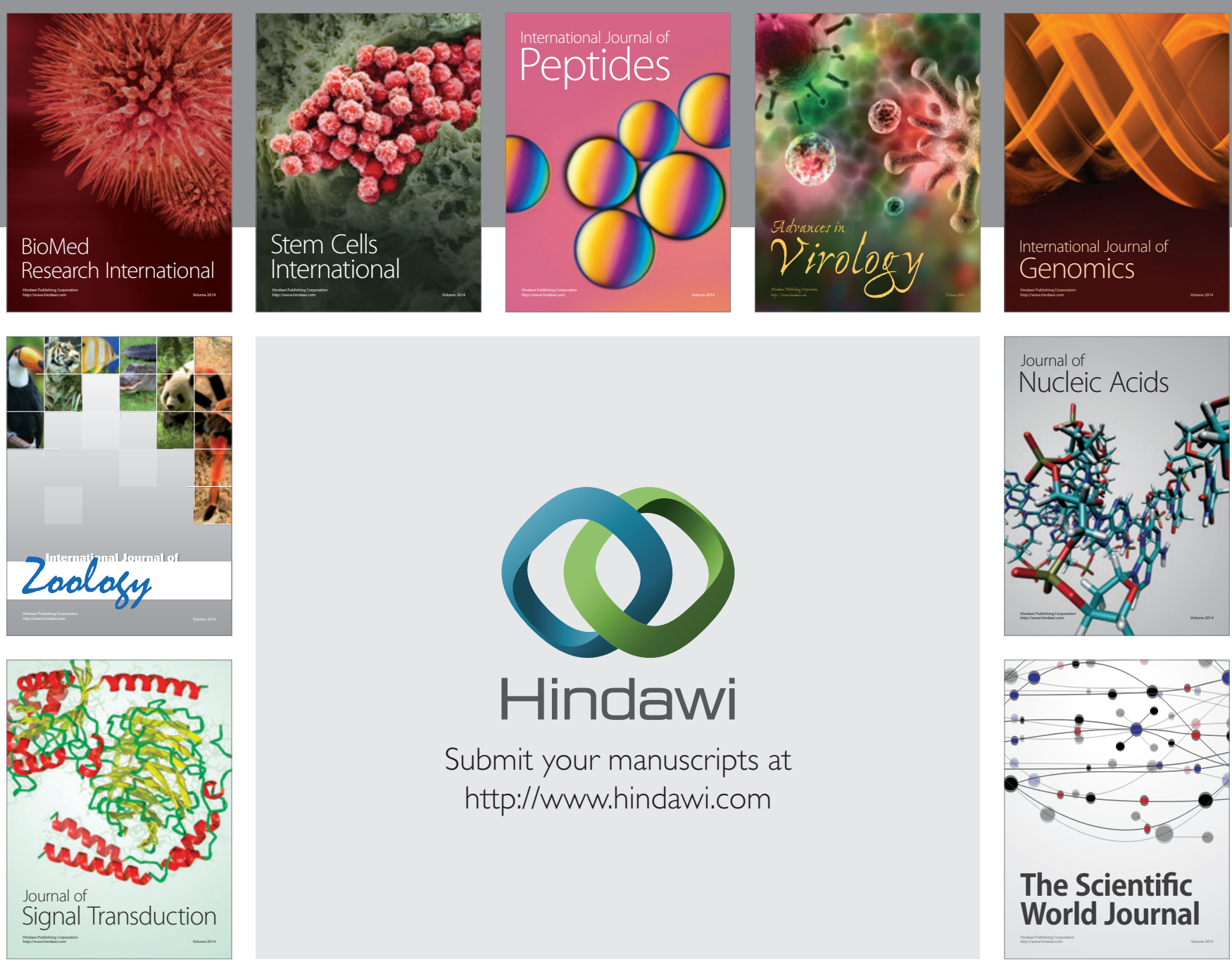

Submit your manuscripts at

http://www.hindawi.com
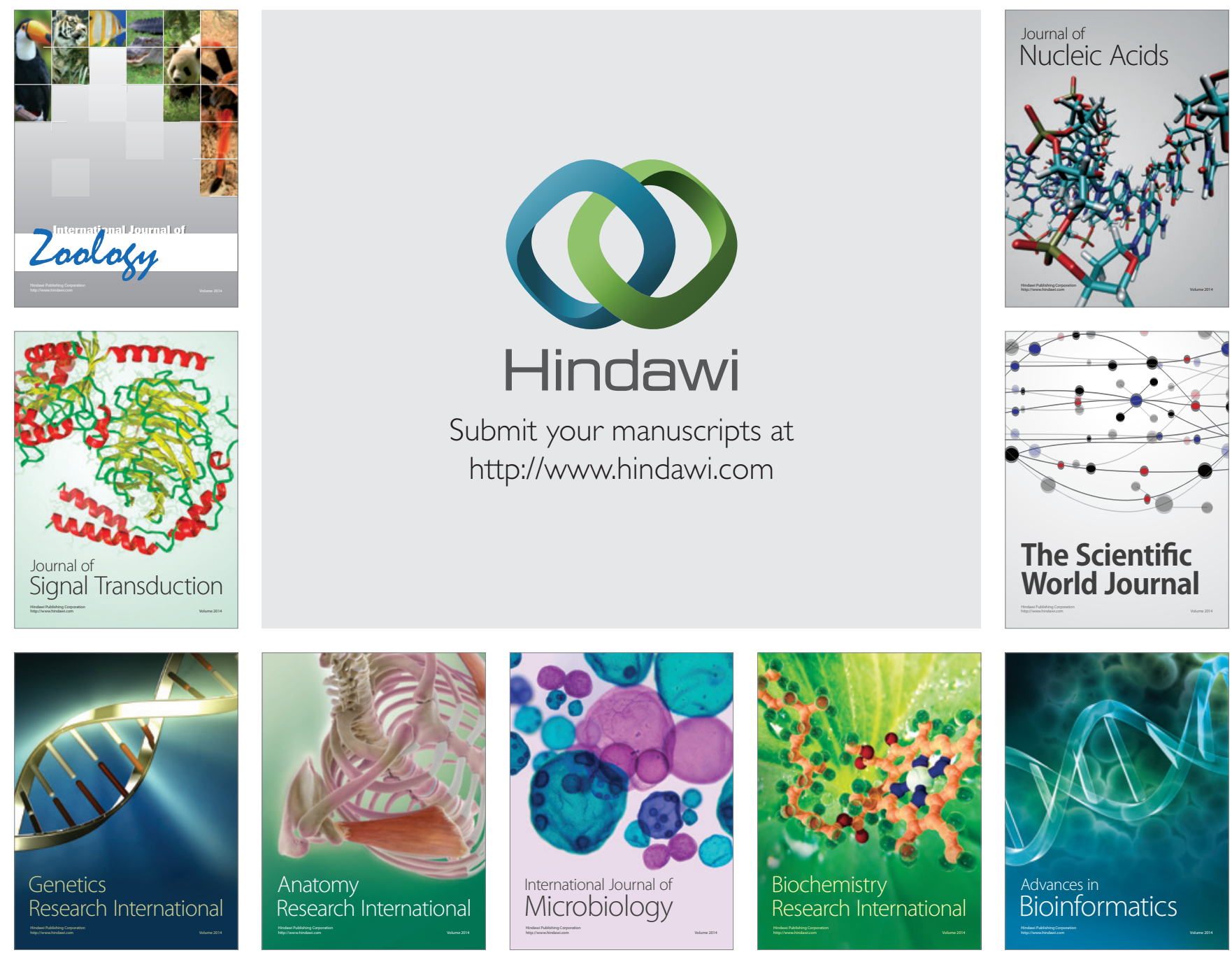

The Scientific World Journal
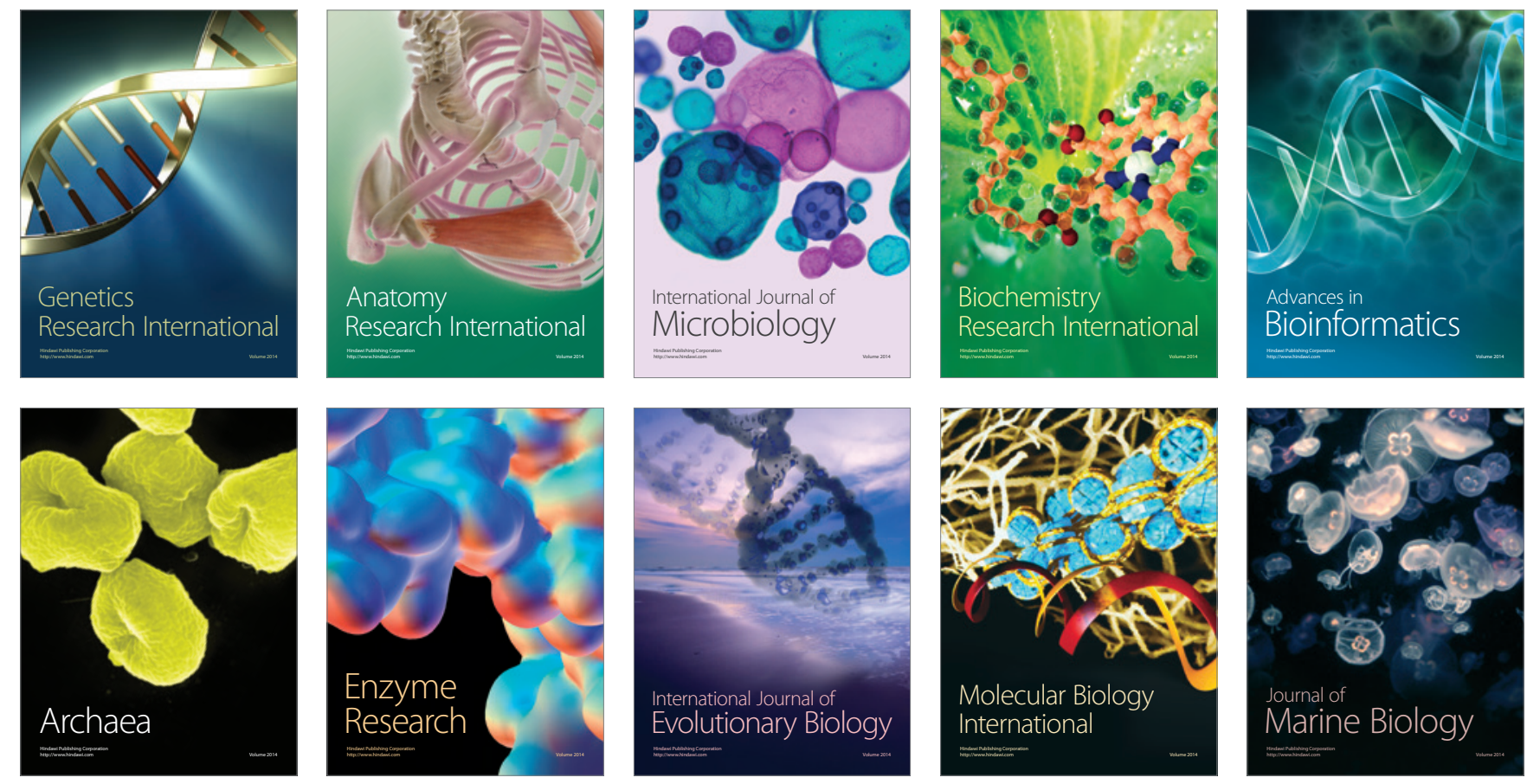